University of Wollongong

Research Online

Australian Institute for Innovative Materials -

Papers

Australian Institute for Innovative Materials

2009

Crystal structure and electronic and thermal properties of TbFeAs00.85

N Kaurav

National Dong Hwa University Taiwan

Y.T Chung

National Dong Hwa University Taiwan

Y.K Kuo

National Dong Hwa University Taiwan

R S Liu

National Taiwan University

T S Chan

National Synchrotron Radiation Research Center, Taiwan

See next page for additional authors

Follow this and additional works at: https://ro.uow.edu.au/aiimpapers

Part of the Engineering Commons, and the Physical Sciences and Mathematics Commons

Research Online is the open access institutional repository for the University of Wollongong. For further information contact the UOW Library: research-pubs@uow.edu.au 


\title{
Crystal structure and electronic and thermal properties of TbFeAsO0.85
}

\begin{abstract}
The crystal structure and the electronic and thermal properties of a high-quality polycrystalline $\mathrm{TbFeAsO}_{0.85}$ sample made by a high-pressure technique are investigated. The crystal structure, as determined by synchrotron X-ray powder diffraction, possesses a tetragonal unit cell (space group: $P 4 / \mathrm{nmm}$ ) with lattice parameters of $a=b=3.8851 \AA$ and $c=8.3630 \AA$. In order to elucidate the electronic structure and oxidation states of corresponding elements, $X$-ray absorption near-edge structure (XANES) spectra are presented. The XANES spectra confirm that the oxidation states of $\mathrm{Fe}, \mathrm{As}$, and $\mathrm{Tb}$ in the TbFeAsO 0.85 sample are $\sim \mathrm{Fe}^{2+}, \sim \mathrm{As}^{3-}$, and $\sim \mathrm{Tb}^{3+}$, respectively, which are consistent with the previously reported band structure calculations. The $n$-type character of the charge carriers as revealed from XANES spectra is corroborated by the negative sign of the Seebeck coefficient (S) in the present study. The heat capacity $\left(C_{P}\right)$ measurement shows an anomaly in the vicinity of the superconducting transition temperature $\left(T_{C}=42.5 \mathrm{~K}\right)$, which confirms the bulk nature of the superconductivity in this material.
\end{abstract}

\section{Keywords}

Crystal, structure, electronic, thermal, properties, TbFeAsO0

\section{Disciplines}

Engineering | Physical Sciences and Mathematics

\section{Publication Details}

Kaurav, N, Chung, Y, Kuo, Y, Liu, R, Chan, T, Chen, J, Lee, J, Sheu, H, Wang, P, Dou, SX, Lee, S, Shi, Y, Belik, A, Yamaura, K \& Takayama-Muromachi, E (2009), Crystal structure and electronic and thermal properties of TbFeAs00.85, Applied Physics Letters, 94(19), pp. 192507-1-192507-3.

\section{Authors}

N Kaurav, Y.T Chung, Y.K Kuo, R S Liu, T S Chan, J.M Chen, J.F Lee, H.S Sheu, Xiaolin Wang, S X. Dou, Sung-lk Lee, Y.G Shi, A.A Belik, K Yamaura, and E Takayama-Muromachi 


\title{
Crystal structure and electronic and thermal properties of $\mathrm{TbFeAsO}_{0.85}$
}

\author{
N. Kaurav, ${ }^{1}$ Y. T. Chung, ${ }^{1}$ Y. K. Kuo, ${ }^{1, a)}$ R. S. Liu, ${ }^{2, a)}$ T. S. Chan, ${ }^{3}$ J. M. Chen, ${ }^{3}$ J.-F. Lee, ${ }^{3}$ \\ H.-S. Sheu, ${ }^{3}$ X. L. Wang, ${ }^{4}$ S. X. Dou, ${ }^{4}$ S. I. Lee, ${ }^{5}$ Y. G. Shi, ${ }^{6}$ A. A. Belik, ${ }^{6}$ K. Yamaura, ${ }^{6}$ \\ and E. Takayama-Muromachi ${ }^{6}$ \\ ${ }^{1}$ Department of Physics, National Dong Hwa University, Hualien 97401, Taiwan \\ ${ }^{2}$ Department of Chemistry, National Taiwan University, Taipei 106, Taiwan \\ ${ }^{3}$ National Synchrotron Radiation Research Center, Hsinchu 300, Taiwan \\ ${ }^{4}$ Institute for Superconducting and Electronic Materials, University of Wollongong, \\ New South Wales 2522, Australia \\ ${ }^{5}$ National Creative Research Initiative Center for Superconductivity, Department of Physics, \\ Sogang University, Seoul 121742, Republic of Korea \\ ${ }^{6}$ National Institute for Materials Science, Tsukuba, Ibaraki 305-0044, Japan
}

(Received 28 December 2008; accepted 24 April 2009; published online 15 May 2009)

The crystal structure and the electronic and thermal properties of a high-quality polycrystalline $\mathrm{TbFeAsO}_{0.85}$ sample made by a high-pressure technique are investigated. The crystal structure, as determined by synchrotron $\mathrm{X}$-ray powder diffraction, possesses a tetragonal unit cell (space group: $P 4 / \mathrm{nmm}$ ) with lattice parameters of $a=b=3.8851 \AA$ and $c=8.3630 \AA$. In order to elucidate the electronic structure and oxidation states of corresponding elements, X-ray absorption near-edge structure (XANES) spectra are presented. The XANES spectra confirm that the oxidation states of $\mathrm{Fe}, \mathrm{As}$, and $\mathrm{Tb}$ in the $\mathrm{TbFeAsO}_{0.85}$ sample are $\sim \mathrm{Fe}^{2+}, \sim \mathrm{As}^{3-}$, and $\sim \mathrm{Tb}^{3+}$, respectively, which are consistent with the previously reported band structure calculations. The $n$-type character of the charge carriers as revealed from XANES spectra is corroborated by the negative sign of the Seebeck coefficient $(S)$ in the present study. The heat capacity $\left(C_{P}\right)$ measurement shows an anomaly in the vicinity of the superconducting transition temperature $\left(T_{c}=42.5 \mathrm{~K}\right)$, which confirms the bulk nature of the superconductivity in this material. () 2009 American Institute of Physics.

[DOI: $10.1063 / 1.3136764]$

Layered rare-earth metal oxypnictides ( $\mathrm{LaFeAsO})$ have emerged as materials of exceptional scientific interest due to the discovery of high-temperature superconductivity in this family. ${ }^{1}$ The strong interplay between structural, magnetic, and superconducting properties in this class of materials has led to the closing of comparisons with the physics of superconducting copper oxides. ${ }^{2,3}$ It has been also recognized that FeAs-based compounds could be a promising thermoelectric candidate in refrigeration applications around liquid nitrogen temperature. ${ }^{4}$ These aspects have motivated several studies replacing La atoms by other lanthanide atoms, resulting in elevated critical temperatures $\left(T_{c}\right)$ from 26 to $56 \mathrm{~K}$, the highest reported so far. ${ }^{5,6}$ These quaternary superconductors have a rather simple structure, consisting of alternating $\left[\mathrm{Fe}_{2} \mathrm{As}_{2}\right]^{2-}$ and $\left[\mathrm{Ln}_{2} \mathrm{O}_{2}\right]^{2-}$ layers with eight atoms in a tetragonal unit cell $(\mathrm{P} 4 / \mathrm{nmm})$, where the $\left[\mathrm{Fe}_{2} \mathrm{As}_{2}\right]^{2-}$ layer is thought to be responsible for superconductivity, while the $\left[\mathrm{Ln}_{2} \mathrm{O}_{2}\right]^{2-}$ layer provides charge carriers, resembling the similar layered structure found in high- $T_{c}$ cuprates. $^{7}$

Superconductivity in $\mathrm{LaFeAsO}$ has been also realized by doping with $\mathrm{F}$ atoms or by producing oxygen vacancies instead, which can create more carriers in the charge reservoir layer. ${ }^{8,9}$ Thus, the stacking of these layers and the ionic states of constituent atoms both play decisive roles in the transport and superconducting properties. Understanding the structural, electronic, and thermal properties would be an important step toward realizing the proposed technological scenario for this class of materials. Although the electrical and

\footnotetext{
a) Authors to whom correspondence should be addressed. Electronic addresses: ykkuo@mail.ndhu.edu.tw and rsliu@ntu.edu.tw.
}

magnetic properties (with $T_{c}=42 \mathrm{~K}$ ) of polycrystalline $\mathrm{TbFeAsO}_{085}$ samples have been described elsewhere recently, ${ }^{9,10}$ in this letter, we focus on the electronic structure and oxidation states by measuring X-ray absorption nearedge structure (XANES) spectra. Moreover, we have independently confirmed the value of $T_{c}$ from a heat capacity anomaly, which is consistent with the previously reported resistivity results. ${ }^{9}$ The thermal conductivity and Seebeck coefficient of $\mathrm{TbFeAsO}_{0.85}$ are also discussed. Our results indicate that the anomalous behavior of the Seebeck coefficient cannot be accounted for by the conventional metallic picture alone.

The polycrystalline $\mathrm{TbFeAsO}_{0.85}$ used in this work was prepared by a high-pressure method. ${ }^{9,10}$ Synchrotron x-ray diffraction (XRD) patterns were recorded at the BL01C2 beam line of the National Synchrotron Radiation Research Center (NSRRC). Structural refinements were carried out by using the General Structural Analysis System (GSAS) program. ${ }^{11}$ The XRD pattern of $\mathrm{TbFeAsO}_{0.85}$ can be indexed based on a tetragonal unit cell (space group: $P 4 / \mathrm{nmm}$ ) with lattice parameters $a=b=3.8851 \AA$ and $c=8.3630 \AA$, similar to those of the $\mathrm{ReFeAsO}_{1-\delta}(\mathrm{Re}=$ rare-earth metal) samples in Ref. 8.

The $\mathrm{Fe}$, As $K$-edge, and $\mathrm{Tb} L_{3}$-edge XANES spectra were recorded in transmission mode at a wiggler BL17C beamline. The $\mathrm{O} K$-edge XANES measurements were performed with a $6 \mathrm{~m}$ high-energy spherical grating monochromator BL20A beamline in the fluorescence-yield mode. Seebeck coefficient and thermal conductivity measurements were simultaneously performed by means of a heat pulse technique. Detailed descriptions of the experimental tech- 

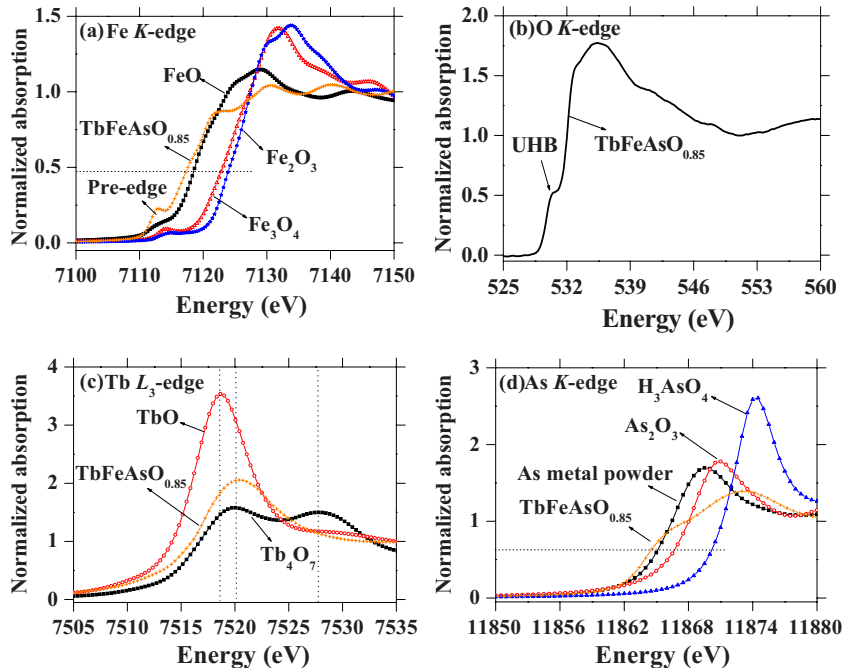

FIG. 1. (Color online) (a) $\mathrm{Fe} K$-edge XANES spectra of $\mathrm{TbFeAsO}_{0.85}$, along with three standards: $\mathrm{FeO}\left(\mathrm{Fe}^{2+}\right), \mathrm{Fe}_{3} \mathrm{O}_{4}\left(\mathrm{Fe}^{2.67+}\right)$, and $\mathrm{Fe}_{2} \mathrm{O}_{3}\left(\mathrm{Fe}^{3+}\right)$. The dashed line at the absorption coefficient value of 0.5 is included to elucidate the chemical shift. (b) $\mathrm{O} K$-edge XANES spectrum of $\mathrm{TbFeAsO}_{0.85}$. (c) $\mathrm{Tb} L_{3}$-edge XANES spectra of $\mathrm{TbFeAsO}_{0.85}$, with two standards: $\mathrm{TbO}$ $\left(\mathrm{Tb}^{2+}\right)$ and $\mathrm{Tb}_{4} \mathrm{O}_{7}\left(\mathrm{~Tb}^{3+}\right.$ and $\mathrm{Tb}^{4+}$ mixture). (d) As $K$-edge XANES spectra of $\mathrm{TbFeAsO}_{0.85}$ with three standards: As metal powder $\left(\mathrm{As}^{0+}\right), \mathrm{As}_{2} \mathrm{O}_{3}$ $\left(\mathrm{As}^{3+}\right)$, and $\mathrm{H}_{3} \mathrm{AsO}_{4}\left(\mathrm{As}^{5+}\right)$.

niques for thermal measurements can be found elsewhere. ${ }^{12}$

The $\mathrm{Fe} K$-edge XANES spectra of $\mathrm{TbFeAsO}_{0.85}$, along with three standards, $\mathrm{FeO}\left(\mathrm{Fe}^{2+}\right), \mathrm{Fe}_{3} \mathrm{O}_{4}\left(\mathrm{Fe}^{2.67+}\right)$, and $\mathrm{Fe}_{2} \mathrm{O}_{3}$ $\left(\mathrm{Fe}^{3+}\right)$, are shown in Fig. 1(a). It is well known that the chemical shift of the main absorption edge to lower energies with decreasing valence of transition metals is a powerful tool for probing the unknown valence of a transition metal. ${ }^{13}$ In Fig. 1(a), a dashed line at the absorption coefficient value of 0.5 is included to elucidate the chemical shift. The $\mathrm{TbFeAsO}_{0.85}$ spectrum is close to, but slightly shifted to, lower energies relative to the $\mathrm{FeO}\left(\mathrm{Fe}^{2+}\right)$ spectrum. This evidence indicates that the electron configuration of $\mathrm{Fe}$ in the $\mathrm{TbFeAsO}_{0.85}$ sample is a mixture of the basic $d^{6}\left(\mathrm{Fe}^{2+}\right)$ state and the $d^{7}$ (plus ligand hole) configuration in the ground state. Moreover, the pre-edge peak at around $7112 \mathrm{eV}$ represents the $1 s \rightarrow 3 d$ transition, which is a dipole forbidden process. Such a pre-edge feature in $\mathrm{TbFeAsO}_{0.85}$ is much stronger than in other standard compounds due to the local tetragonal ligand field allowing dipole transitions into $3 d$ related states. $^{13}$ The $\mathrm{O} K$-edge XANES spectrum of $\mathrm{TbFeAsO}_{0.85}$ is displayed in Fig. 1(b). The appearance of the broad peak located at about $530 \mathrm{eV}$ indicates the $n$-type character of the charge carriers. Such an observation is also reported for other $n$-type high- $T_{c}$ superconductive copper oxide $(\mathrm{Nd}, \mathrm{Ce})_{2} \mathrm{CuO}_{4+\delta}$ systems, which is ascribed to transitions into the $\mathrm{O} 2 p$ states hybridized with the upper Hubbard band (UHB). ${ }^{14,15}$

In Fig. 1(c), $\mathrm{Tb} L_{3}$-edge XANES spectra of $\mathrm{TbFeAsO}_{0.85}$ and two standard $\mathrm{TbO}\left(\mathrm{Tb}^{2+}\right)$ and $\mathrm{Tb}_{4} \mathrm{O}_{7}\left(\mathrm{~Tb}^{3+}\right.$ and $\mathrm{Tb}^{4+}$ mixture) compounds are shown. It is well known that the $\mathrm{Tb}_{4} \mathrm{O}_{7}$ spectrum has two broad peaks centered at $\sim 7522$ and $\sim 7530 \mathrm{eV}$, reflecting the inhomogeneous, mixed-valence nature of the sample. ${ }^{16}$ The peak position at $\sim 7522 \mathrm{eV}$ in the $\mathrm{Tb} L_{3}$-edge XANES spectrum of $\mathrm{TbFeAsO}_{0.85}$, together with the absence of a second absorption peak, indicates that the $\mathrm{Tb}$ is neither a tetravalent nor a mixed valence but is instead essentially $\mathrm{Tb}^{3+}$ in this sample. Figure 1(d) shows the

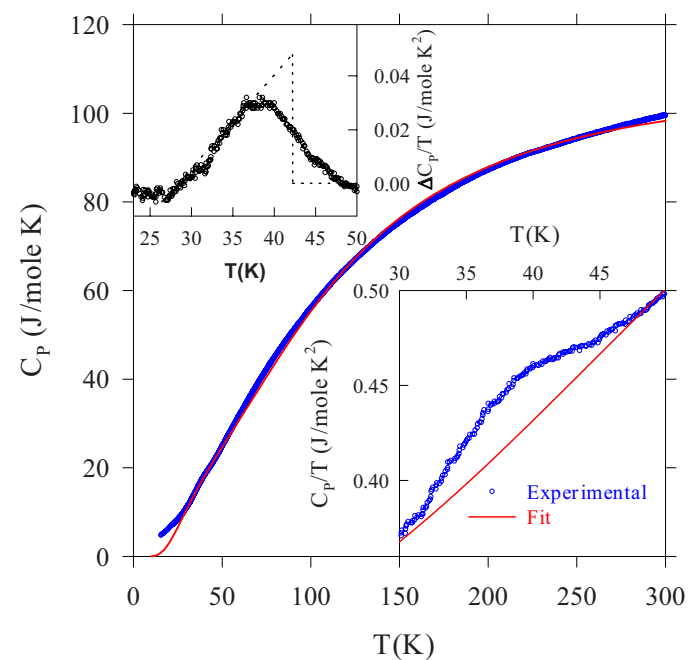

FIG. 2. (Color online) The temperature dependence of the specific heat for $\mathrm{TbFeAsO}_{0.85}$. The solid red line represents the fit using the Einstein model. The lower and upper insets show the plots of $C p / T$ vs $T$ and $\Delta C p / T$ vs $T$, respectively. The dashed line in the lower inset shows the Debye model fit.

As $K$-edge XANES spectrum of the $\mathrm{TbFeAsO}_{0.85}$ sample with three standards, including As powder $\left(\mathrm{As}^{0}\right), \mathrm{As}_{2} \mathrm{O}_{3}$ $\left(\mathrm{As}^{3+}\right)$, and $\mathrm{H}_{3} \mathrm{AsO}_{4}\left(\mathrm{As}^{5+}\right)$. In view of the absorption coefficient value of $\sim 0.5$, the As $K$-edge spectrum of $\mathrm{TbFeAsO}_{0.85}$ is clearly shifted to lower energies relative to that of As powder, indicating that the oxidation number of As in $\mathrm{TbFeAsO}_{0.85}$ is negative. Considering the valence of $\mathrm{Fe}$ and $\mathrm{Tb}$, we assume that the oxidation state of $\mathrm{As}$ in $\mathrm{TbFeAsO}_{0.85}$ is approximately $3^{-}$.

In Fig. 2 we show the specific heat $\left(C_{P}\right)$ of $\mathrm{TbFeAsO}_{0.85}$ in the temperature range of $20-300 \mathrm{~K}$. The observed room temperature value is $C_{P}(300 \mathrm{~K}) \approx 100 \mathrm{~J} / \mathrm{mol} \mathrm{K}$, which is close to the Dulong-Petit limiting value and is consistent with previous results. ${ }^{17-19}$ This signifies that the bulk of the contribution indeed comes from the vibrational heat capacity in this temperature range. The solid line in Fig. 2 represents a fitting of the $C_{P}$ using the Einstein model, $C_{E}$ $=3 r R \Sigma_{i} a_{i}\left[x_{i}^{2} e^{x_{i}} /\left(e^{x_{i}}-1\right)^{2}\right]$, where $x_{i}=h \nu_{E} / k_{B} T_{i}$. Three optical phonons $(i=1,2,3)$ having the Einstein frequencies $h \nu_{E} / k_{B}$ $=122,330$, and $430 \mathrm{~K}$ and relative occupations $a_{i}$ were used. It is interesting to note that our phonon frequencies are nicely matched with those seen in a recent Raman measurement ${ }^{20}$ and in lattice dynamical calculations. ${ }^{21,22}$

Since the Debye model is more applicable in the lowtemperature regime, we have further estimated the specific heat between 20 and $60 \mathrm{~K}$ with a cutoff frequency given by the Debye temperature $\theta_{D}=330 \mathrm{~K}$, and this is plotted as a dashed line in the lower inset of Fig. 2. The deduced value of the Debye temperature is comparable with reported data. ${ }^{17-19,22}$ The most apparent feature in our $C_{P}$ result is that an abnormality of the heat capacity is clearly observed in the vicinity of the superconducting transition temperature $\left(T_{c}\right)$, which has been absent in earlier reports on FeAs-based superconductors so far. ${ }^{17-19}$ The change in the entropy, $\Delta S$ $=0.31 \mathrm{~J} / \mathrm{mol} \mathrm{K}$, associated with the superconducting transition was then obtained by subtracting the lattice part from the experimental data. In the upper inset of Fig. $2, \Delta C_{P} / T$ is plotted as a function of $T$ within a narrow temperature interval of $25-50 \mathrm{~K}$ at around $T_{c}$. Here, the excess heat capacity $(\Delta C)$ is obtained by subtracting the estimated background 


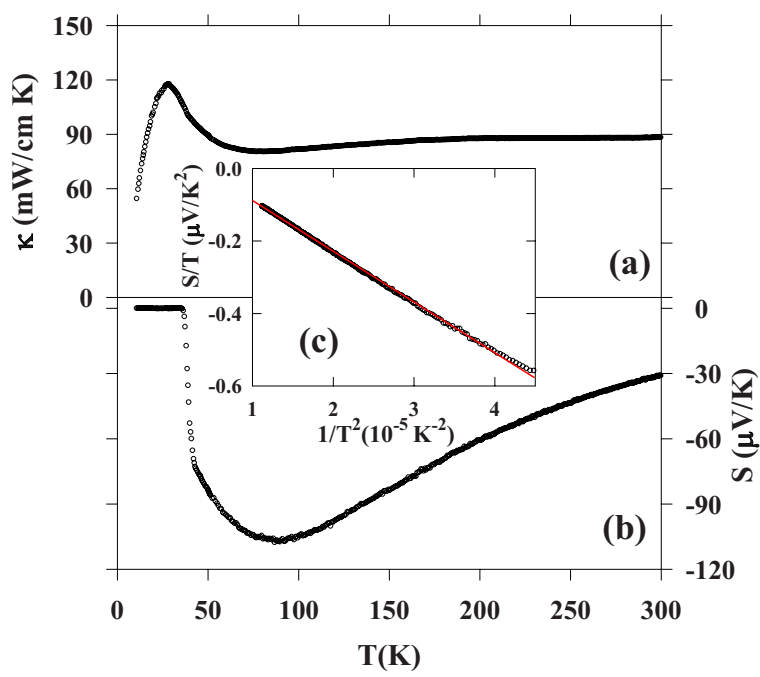

FIG. 3. (Color online) (a) Thermal conductivity vs temperature for $\mathrm{TbFeAsO}_{0.85}$. (b) Seebeck coefficient as a function of temperature for $\mathrm{TbFeAsO}_{0.85}$. (c) $S / T$ is found to be linear with $1 / T^{2}$ above $150 \mathrm{~K}$.

from the total measured $C_{P}$. By considering the entropy conservation between the superconducting and normal states, we have deduced that the mean field heat capacity jump has a value of $2.2 \mathrm{~J} / \mathrm{mol} \mathrm{K}$ and that the superconducting transition $T_{c}=42.5 \mathrm{~K}$, which is consistent with resistivity measurements. ${ }^{9}$

In Fig. 3, we show the variations of the Seebeck coefficient $S(T)$ and the thermal conductivity $\kappa(T)$ with temperature for $\mathrm{TbFeAsO}_{0.85}$. The negative sign of $S$ is consistent with the electronlike conduction mechanism. The absolute value of $S$ increases from $31 \mu \mathrm{V} / \mathrm{K}$ at room temperature to a maximum value $\left(S_{\max }\right)$ of $107 \mu \mathrm{V} / \mathrm{K}$ at $90 \mathrm{~K}$ and then decreases sharply toward zero when the temperature reaches $T_{c}$ [Fig. 3(b)]. The temperature variation and absolute value of $S$ are similar to those reported for iron-based superconductors. ${ }^{18,19,23,24}$ It should be pointed out here that these materials exhibit an exceptionally large $S$ and a rather low electrical resistivity at low temperatures, which is thought to be advantageous for low-temperature refrigeration applications. ${ }^{4}$ We have tried to fit our experimental results for the conventional diffusion and phonon drag component to $S$, written as $(a T+b / T)$, as is applicable to high- $T_{C}$ cuprates. ${ }^{25}$ In Fig. 3(c), it can be seen that $S / T$ is linear with $1 / T^{2}$ above $150 \mathrm{~K}$, and there is a departure from a simple metallic behavior below this temperature. It has been argued that the freezing-out of Umklapp processes could have resulted in the dome-shaped feature in $S(T)$ and the enhancement of $S$ in the case of high- $T_{c}$ cuprates. ${ }^{25}$ The effects of large anisotropy and the shape of the Fermi surface are expected to have an impact on the population of phonon modes and, hence, Umklapp processes. ${ }^{22}$ Also, the appearance of a sharp low-temperature peak in the thermal conductivity data [Fig. 3(a)] further supports the necessity of an Umklapp process in the thermal transport. However, strong electron correlations and spin fluctuations should suppress the nonequilibrium effects in the phonon distribution function, resulting in a significant slackening of the phonon drag term. The band structure value of $S$ of about $6.8 \mu \mathrm{V} / \mathrm{K}$ provides additional arguments against the conventional metallic interpretation of $S .22$
On the other hand, spin entropy could be another likely source of the anomalously large $S$ in the FeAs-based superconductors. It is worth mentioning that the importance of spin entropy has been pointed out for large $S$ in the $\mathrm{Na}_{x} \mathrm{CoO}_{2}$ system. ${ }^{26}$ This indicates that a large absolute value of $S$ can be realized when the thermal energy $\left(k_{B} T\right)$ is much less than the on-site Coulomb repulsion $U$, and $S$ can be approximately $\sim-k_{B} \ln 2 /|e|$. Here, the $\ln 2$ term comes from the spin fluctuations of $\mathrm{Fe}^{+2}$ ions, and the estimated value of $|S| \sim 60 \mu \mathrm{V} / \mathrm{K}$ could account for about $60 \%$ of the observed $S_{\max }$.

In summary, a high-quality polycrystalline $\mathrm{TbFeAsO}_{0.85}$ sample was synthesized by a high-pressure method, and the crystal structure and the electronic and thermal properties were investigated. The XANES spectra confirm that the oxidation state of $\mathrm{Fe}$, $\mathrm{As}$, and $\mathrm{Tb}$ in the $\mathrm{TbFeAsO}_{0.85}$ sample is $\sim \mathrm{Fe}^{2+}, \sim \mathrm{As}^{3-}$, and $\sim \mathrm{Tb}^{3+}$, respectively. The specific heat measurement shows an anomaly in the vicinity of the superconducting transition temperature $\left(T_{c}=42.5\right)$, which confirms the bulk nature of the superconductivity in this material. The negative sign of the measured Seebeck coefficient signifies electronlike charge carriers, in good agreement with the XANES measurements. Our results indicate that the large $S$ value cannot be explained by the conventional metallic picture alone, and spin entropy could be another likely source for the observed large Seebeck coefficient in FeAs-based superconductors.

The authors would like to thank the National Science Council of Taiwan for financially supporting this research under Contract Nos. NSC 97-2628-M-259-001-MY3 (Y.K.K) and NSC 97-2113-M-002-012-MY3 (R.S.L). This work is also partially supported by the Australian Research Council through a Discovery project, DP0558753, the Japan Science and Technology Agency (JST), TRIP of Japan, and the program of Accelerated Research of the Korea Science and Engineering Foundation of the Ministry of Science and Technology (MOST/KOSEF) of Korea.

\footnotetext{
${ }^{1}$ Y. Kamihara et al., J. Am. Chem. Soc. 130, 3296 (2008).

${ }^{2} \mathrm{X}$. H. Chen et al., Nature (London) 453, 761 (2008).

${ }^{3}$ T. Y. Chen et al., Nature (London) 453, 1224 (2008).

${ }^{4}$ L. Pinsard-Gaudart et al., Phys. Status Solidi (RRL) 2, 185 (2008).

${ }^{5}$ C. Wang et al., Europhys. Lett. 83, 67006 (2008).

${ }^{6}$ R. H. Liu et al., Phys. Rev. Lett. 101, 087001 (2008).

${ }^{7}$ P. Quebe et al., J. Alloys Compd. 302, 70 (2000).

${ }^{8}$ Z. A. Ren et al., Europhys. Lett. 83, 17002 (2008).

${ }^{9}$ Y. G. Shi et al., J. Phys. Soc. Jpn. Suppl. C 77, 155 (2008).

${ }^{10}$ Y. G. Shi et al., arXiv:0812.4907.

${ }^{11}$ A. C. Larson and R. B. V. Dreele, "Generalized Structure Analysis System," Los Alamos National Laboratory Report No. LAUR 86-748, 1994.

${ }^{12}$ Y. K. Kuo et al., Phys. Rev. B 64, 125124 (2001)

${ }^{13}$ J. Wong et al., Phys. Rev. B 30, 5596 (1984).

${ }^{14}$ E. Pellegrin et al., Phys. Rev. B 47, 3354 (1993).

${ }^{15}$ R. S. Liu et al., Solid State Commun. 118, 367 (2001).

${ }^{16}$ U. Staub et al., Phys. Rev. B 50, 7085 (1994).

${ }^{17}$ Y. Kohama et al., Phys. Rev. B 78, 020512 (2008).

${ }^{18}$ A. S. Sefat et al., Phys. Rev. B 78, 104505 (2008).

${ }^{19}$ M. A. McGuire et al., Phys. Rev. B 78, 094517 (2008)

${ }^{20}$ V. G. Hadjiev et al., Phys. Rev. B 77, 220505 (2008).

${ }^{21}$ L. Boeri et al., Phys. Rev. Lett. 101, 026403 (2008).

${ }^{22}$ D. J. Singh et al., Phys. Rev. Lett. 100, 237003 (2008).

${ }^{23}$ L. J. Li et al., Phys. Rev. B 78, 132506 (2008).

${ }^{24}$ A. S. Sefat et al., Phys. Rev. B 77, 174503 (2008).

${ }^{25}$ J. L. Cohn et al., Phys. Rev. Lett. 66, 1098 (1991).

${ }^{26}$ Y. Wang et al., Nature (London) 423, 425 (2003).
} 